\title{
AUTOEVALUACIÓN Y COEVALUACIÓN DE LA PRÁCTICA DOCENTE DEL PROFESORADO UNIVERSITARIO
}

Self-evaluation and peer evaluation of lecturers' teaching performance

Autoavaliação e coavaliação da prática docente dos professores universitários

\author{
M. Inmaculada González-Alonso (1) \\ Raquel Domínguez-Fernández (2) \\ Ángeles Diez-Fernández (3) \\ Ángel Pérez-Pueyo (4) \\ Carlos Gutiérrez-García (5)
}

Apoyo/financiación del trabajo. Convocatoria del Plan de Apoyo a los Grupos de Innovación Docente 2017 de la Universidad de León. (PAGID 2017)

(1) Universidad de León, España. Teléfono: +34 987291049. Correo electrónico: igonaf@unileon.es

(2) Universidad de León, España. Teléfono: +34 987291049. Correo electrónico: rdomf@unileon.es

(3) Universidad de León, España. Teléfono: +34 987293030. Correo electrónico: madief@unileon.es

(4) Universidad de León, España. Teléfono: +34 987293024. Correo electrónico: angel.perez.pueyo@unileon.es

(5) Universidad de León, España. Teléfono: +34 987293058. Correo electrónico: carlos.gutierrez@unileon.es

\section{Resumen}

El objetivo de esta experiencia fue implementar un sistema de evaluación formativa que permitiese a los profesores universitarios implicados desarrollar un proceso de reflexión crítica sobre sus propias actuaciones docentes (autoevaluación) antes y después de recibir el feedback de sus colegas (co-evaluación). Se realizaron observaciones sin intervención durante 6 sesiones de una hora utilizando un registro de observación en 6 asignaturas. Para los docentes evaluados, todos ellos expertos, el implicarse en procesos de autoevaluación y, sobre todo, el diálogo entre pares supone un estímulo a la reflexión (auto)crítica y al crecimiento profesional. En el futuro se propone ampliar esta experiencia en el número de observadores y los tiempos de observación. Por último, entendemos que este tipo de evaluación potenciaría su valor formativo si pudiese transferirse a profesores noveles interesados en la mejora de sus competencias docentes. Palabras clave: Autoevaluación; co-evaluación; práctica docente; profesores universitarios 


\begin{abstract}
The objective of this experience was to design a formative evaluation procedure that could enable the lecturers involved to develop a process of critical reflection on their own teaching performance (self-evaluation) before and after having received their peers' feedback. Six sessions belonging to six subjects were observed and evaluated by using an observation sheet. For the evaluated lecturers participating in self-evaluation processes and, above all, in peer dialogues, has been very positive since it has stimulated their critical reflection and professional growth. Suggestions for improvement of this experience would include an increase in the observers' number and the observation time. This kind of evaluation would also enhance its formative value if transferred to novel lecturers focusing on the improvement of their teaching competences.
\end{abstract}

Keywords: Self-evaluation; peer evaluation; teaching performance; lecturers

\title{
Resumo
}

Esta experiência teve como objetivo projetar um sistema de avaliação formativa que permitisse aos docentes universitários envolvidos desenvolver um processo de reflexão crítica sobre os seus próprios desempenhos docentes (autoavaliação) antes e depois de receber o feedback dos seus pares (coavaliação). Realizaram-se observações sem intervenção durante seis sessões de uma hora, utilizando uma folha para registar as observações de seis disciplinas. Para os docentes avaliados, todos eles experts, a implicação em processos de autoavaliação e, sobretudo, em diálogos entre pares, pressupõe um estímulo à reflexão (auto)crítica e ao crescimento profissional. Como sugestão futura propõe-se aumentar o número de observadores nesta experiência e o tempo de observação. Além disso, entendemos que este tipo de avaliação aumentaria o seu valor formativo, caso fosse usado com professores novatos, que estejam interessados na melhoria das suas competências docentes.

Palavras-chave: Autoavaliação; coavaliação; prática docente; professores universitários 


\section{Introducción}

La evaluación de la actividad docente en la Enseñanza Superior plantea una serie de preguntas básicas sobre qué se debe valorar, quién debe hacerlo y cómo debe hacerse (De Juan, Pérez, Gómez-Torres, Vizcaya \& Mora, 2007). Los instrumentos utilizados suelen ser las encuestas a los alumnos y a los propios profesores, que aportan cierta información, pero, por su carácter final y cuantitativo, tienen una incidencia mínima en las clases.

Dentro de todos los aspectos susceptibles de ser evaluados en la actividad docente, la actuación docente, es decir, el desarrollo en el aula de los procesos de enseñanza y aprendizaje parece el más difícil de evaluar. Es necesario poner en práctica un sistema de evaluación de la actuación del docente que permita al profesor reflexionar sobre su propia práctica (Brookfield, 1995) e introducir en sus siguientes clases los cambios pertinentes, con el fin de mejorar no solo su propio crecimiento profesional sino también en el aprendizaje de los alumnos.

Nuestro objetivo fue implementar un sistema de evaluación formativa que permitiese a los profesores universitarios implicados desarrollar un proceso de reflexión crítica sobre sus propias actuaciones docentes (autoevaluación) antes y después de recibir el feedback de sus colegas (co-evaluación).

\section{Contextualización}

La experiencia fue llevada a cabo por el grupo IFAHE de la Universidad de León, formado por 8 profesores de 7 áreas de conocimiento (Botánica, Derecho del Trabajo y de la Seguridad Social, Didáctica y Expresión Corporal, Educación Física y Deportiva, Filología Hispánica, Filología Inglesa, Geografía e Ingeniería Eléctrica) y con una experiencia de más de 24 años de media como docentes, 16 años formando formadores y entre 10 y 20 años utilizando la evaluación formativa en sus clases.

\section{Diseño y desarrollo}

Fases previas (1-6) (ver Figura 1). Antes de las observaciones los docentes se habían formado en las características que convierten a la enseñanza universitaria en excelente; se habían definido los criterios, procedimientos e instrumentos para la evaluación de la actividad docente; y, finalmente se les había entrenado en la observación y la evaluación 
de la docencia, tanto la propia (autoevaluación) como la de sus compañeros (coevaluación).

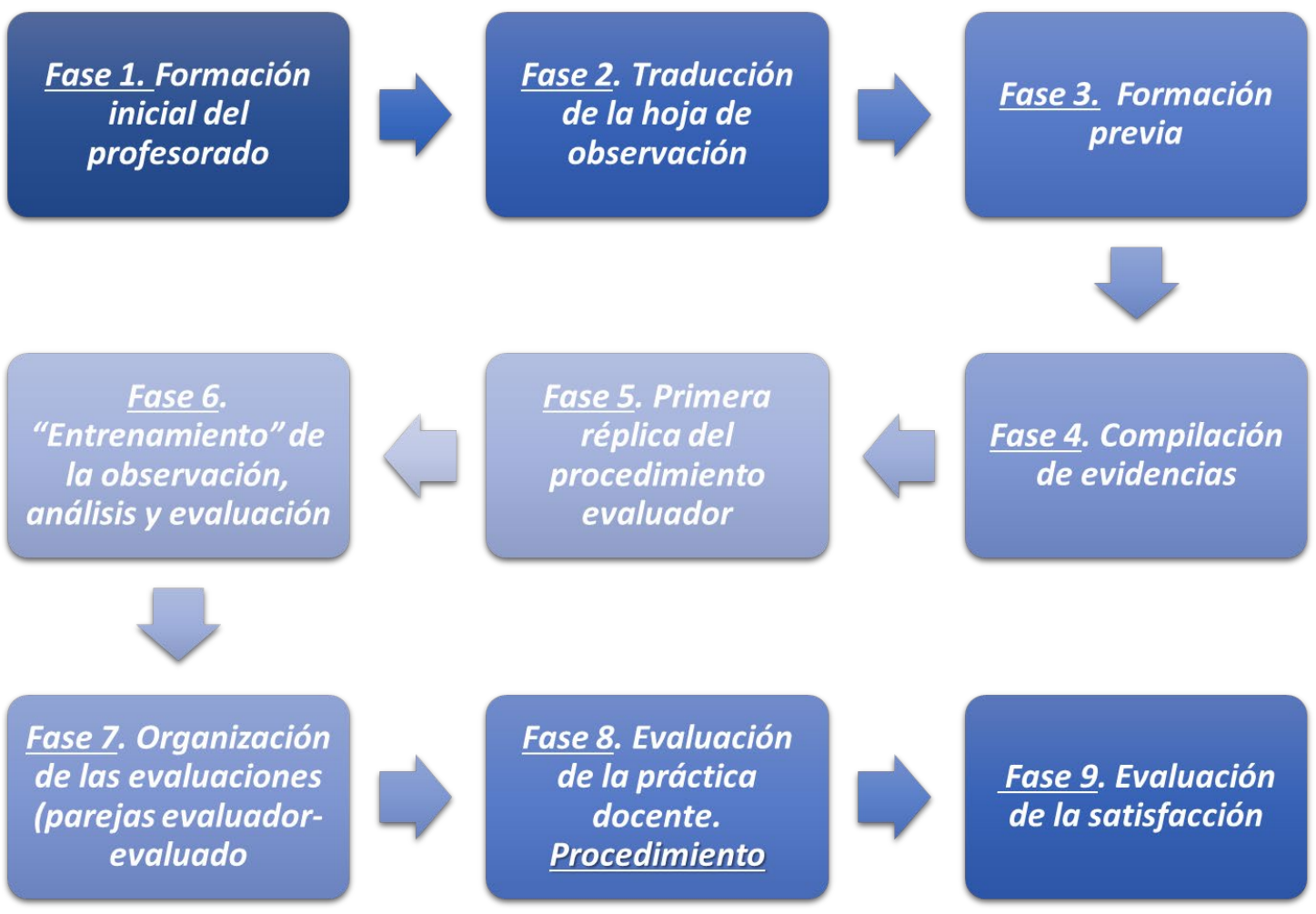

Figura 1. Fases realizadas en la experiencia

Experiencia (fases 7-9) (ver Figuras 1 y 2 ). Se organizaron parejas de docentes observado - observador de áreas de conocimiento diferentes, según su disponibilidad. Posteriormente, se realizaron observaciones sin intervención durante 6 sesiones de una hora utilizando un registro de observación en el que se reflejaron la planificación de la sesión, la autoevaluación previa a la co-evaluación, el feedback del evaluador y el aprendizaje final (conclusiones) del observado. Las asignaturas implicadas pertenecían a 4 grados y 2 másteres: Derecho Social Comunitario y Extranjería (Grado en Relaciones Laborales y Recursos Humanos), Ampliación Deportiva en Judo (Grado en CC de la E.F. y del Deporte), Electrotecnia General (Grado en ingeniería Eléctrica), Enseñanza y aprendizaje de las Ciencias Experimentales II: Biología y Geología (Grado en E. Primaria), Inglés aplicado al entrenamiento y rendimiento deportivo (Máster U. en Entrenamiento y Rendimiento Deportivo) y Prácticum: Módulo Ciencias Sociales (Máster U. en Formación del Profesorado de E. Secundaria). 


\begin{tabular}{|l|}
\hline (1) \\
\hline - Hoja de observación con información previa \\
\hline (2) \\
\hline Durante una hora se tomaron notas de observación \\
\hline (3) \\
\hline - Los docentes observados realizaron reflexiones iniciales AUTOEVALUACIÓN 1 \\
\hline (4) \\
\hline - Los evaluadores realizaron la evaluación de las clases COEVALUACIÓN \\
\hline (5) \\
\hline - Los evaluadores se reunieron con los observados (feedback) COEVALUACIÓN \\
\hline (6) \\
\hline LOS profesores observados reflejan sus reflexiones tras el feedback. AUTOEVALUACIÓN_2 \\
\hline
\end{tabular}

Figura 2. Evaluación de la práctica docente. Fase 8. Detalle COEVALUACIÓN-AUTOEVALUACIÓN

\section{Evaluación y conclusiones}

El análisis de los registros de observación ha permitido descubrir los procesos reflexivos desarrollados por los docentes observados y por sus observadores, una de las competencias que evidencian la excelencia docente (Rolls, 2007; Thorsen \& De Vore, 2013).

Las reflexiones incluidas en las autoevaluaciones previas al feedback de los observadores mostró la satisfacción unánime de los docentes con la metodología que utilizaron durante las sesiones, pero no con su planificación de las mismas. Sus propuestas de mejora para futuras clases se centraron en ajustar más la cantidad de los contenidos a la duración de las clases y atender a la heterogeneidad de sus estudiantes.

Además, una vez discutido el feedback recibido la mitad de los observados explicaron que la coevaluación y la autoevaluación habían coincidido en muchos puntos, en especial en los aspectos positivos de las metodologías. En cuanto a la información no coincidente se refería, sobre todo, al grado de implicación de los alumnos en la clase. El mayor problema que los docentes adujeron fue la dificultad para organizar los horarios de las observaciones y las reuniones posteriores.

En concordancia con la literatura (Anijovich \& Cappelletti, 2018) esta experiencia aunque limitada por el número de profesores implicados - ha mostrado que, como los propios docentes han explicitado, el implicarse en procesos de autoevaluación y, sobre 
todo, el diálogo entre pares supone, para profesores -en este caso expertos- un estímulo a la reflexión (auto)crítica y al crecimiento profesional.

En el futuro se propone ampliar esta experiencia 1) en el número de observadores por sesión, con el fin de que los docentes puedan recibir un feedback más amplio y contrastado y 2) en los tiempos de observación, intentando que, como los propios docentes observados explican, este tipo de práctica se convierta en algo habitual con el fin de "evitar acomodarse y corregir vicios". Por último, entendemos que este tipo de evaluación potenciaría su valor formativo si pudiese transferirse a profesores noveles interesados en la mejora de sus competencias docentes.

\section{Referencias}

Anijovich, R., \& Cappelletti, G. (2018). La práctica reflexiva en los docentes en servicio. Posibilidades y limitaciones. Espacios en Blanco. Revista de Educación, 28, 75-90.

Brookfield, S. (1995) Becoming a Critically Reflective Teacher. San-Francisco: JosseyBass.

De Juan, J., Pérez, R. M., Gómez-Torres, M. J., Vizcaya, M. F., \& Mora, J. M. (2007). Buenas prácticas en la evaluación de la docencia y del profesorado universitario. En A. Martínez \& V. Carrasco (Eds.): La multidimensionalidad de la educación universitaria. Vol. I. (pp. 155-182). Alcoy: Marfil.

Rolls, E. (1997). Competence in professional practice: some issues and concerns. Educational Research, 39 (2), 195-210.

Thorsen, C. A., \& De Voreb, S. (2013). Analyzing reflection on/for action: A new approach Reflective Practice: International and Multidisciplinary Perspective, $14(1), 88-103$ 